\title{
An Essential Residue in the Flexible Peptide Linking the Two Idiosynchratic Domains of Bacterial Tyrosyl-tRNA Synthetases
}

\author{
Carole Gaillard ${ }^{\ddagger}$ and Hugues Bedouelle* \\ Unité de Biochimie Cellulaire, CNRS URA2185, Institut Pasteur, 28 rue Docteur Roux, 75724 Paris Cedex 15, France
}

Received January 31, 2001; Revised Manuscript Received April 4, 2001

\begin{abstract}
Tyrosyl-tRNA synthetase (TyrRS) from Bacillus stearothermophilus comprises three sequential domains: an N-terminal catalytic domain, an $\alpha$-helical domain with unknown function, and a C-terminal tRNA binding domain (residues 320-419). The properties of the polypeptide segment that links the $\alpha$-helical and C-terminal domains, were analyzed by measuring the effects of sequence changes on the aminoacylation of tRNA ${ }^{\text {Tyr }}$ with tyrosine. Mutations F323A (Phe323 into Ala), S324A, and G325A showed that the side chain of Phe323 was essential but not those of Ser324 and Gly325. Insertions of Gly residues between Leu322 and Phe323 and the point mutation L322P showed that the position and precise orientation of Phe323 relative to the $\alpha$-helical domain were important. Insertions of Gly residues between Gly325 and Asp326 and deletion of residues 330-339 showed that the length and flexibility of the sequence downstream from Gly325 were unimportant but that this sequence could not be deleted. Mutations F323A, $-\mathrm{L},-\mathrm{Y}$, and $-\mathrm{W}$ showed that the essential property of Phe323 was its aromaticity. The Phe323 side chain contributed to the stability of the initial complex between TyrRS and tRNA ${ }^{\text {Tyr }}$ for $2.0 \pm 0.2 \mathrm{kcal} \cdot \mathrm{mol}^{-1}$ and to the stability of their transition state complex for $4.2 \pm 0.1 \mathrm{kcal} \cdot \mathrm{mol}^{-1}$, even though it is located far from the catalytic site. The results indicate that the disorder of the $\mathrm{C}$-terminal domain in the crystals of TyrRS is due to the flexibility of the peptide that links it to the helical domain. They identified Phe323 as an essential residue for the recognition of tRNA ${ }^{\text {Tyr }}$.
\end{abstract}

Tyrosyl-tRNA synthetase (TyrRS $)^{1}$ catalyzes the charging of tRNA ${ }^{\text {Tyr }}$ in a two-step reaction. Tyrosine is first activated with ATP to give tyrosyl adenylate (Tyr-AMP) and then transferred to the acceptor end of tRNA ${ }^{\mathrm{Tyr}}$. The crystal structure of TyrRS from Bacillus stearothermophilus has been solved at $2.3 \AA$ resolution (1). It is a homodimeric protein (Figure 1). Each subunit comprises two sequential domains: a well-ordered N-terminal domain (residues 1-319), followed by an apparently disordered C-terminal domain (residues 320-419). The N-terminal domain contains the binding site for Tyr-AMP and the interface of dimerization. It can be divided into two topological subdomains: an $\alpha / \beta$ subdomain (residues 1-220), which contains a six-stranded $\beta$-sheet and whose fold is characteristic of the class I aminoacyl-tRNA synthetases (aaRS), followed by an $\alpha$-helical subdomain (residues 248-319), whose function is unknown $(1,2)$. The $\mathrm{N}$-terminal domain has the same crystal structure in the full-length protein and as a recombinant truncated protein (N-terminal fragment) (3). The N-terminal fragment forms Tyr-AMP with normal kinetics. The C-

* Corresponding author. Tel: 33-1-45 6883 79. Fax: 33-1-40 61 30 43. E-mail: hbedouel@pasteur.fr.

† Present address: Département d'Ingénierie et d'Etude des Protéines, CEA Saclay, 91191 Gif sur-Yvette, France.

${ }^{1}$ Abbreviations: IPTG, isopropyl $\beta$-D-thiogalactoside; NMR, nuclear magnetic resonance; PMSF, phenylmethanesulfonyl fluoride; TyrRS, tyrosyl-tRNA synthetase; TyrRS*, a derivative of TyrRS from Bacillus stearothermophilus carrying the mutation $\mathrm{A} 321 \mathrm{C}$ and a C-terminal extension Leu-Glu-His 6 ; TyrRS $(\Delta 1)$, a derivative of TyrRS from $B$. stearothermophilus carrying a deletion of residues 318-417; Tyr-AMP, tyrosyl adenylate.

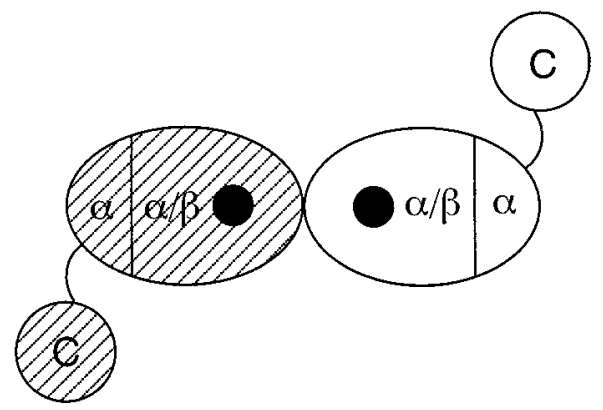

FIGURE 1: Schematic organization of the TyrRS domains. The $\mathrm{N}$-terminal domain (residues $1-319$ ) can be divided into an $\alpha / \beta$ subdomain, which contains the binding site of Tyr-AMP (schematized as a black disk) and the interface of dimerization, followed by an $\alpha$-helical subdomain (residues $248-319$ ). The C-terminal domain, which is involved in the binding of tRNA ${ }^{\text {Tyr }}$, is folded in solution but apparently disordered in the crystals.

terminal domain is necessary for tRNA binding and charging (4).

Biophysical studies in solution have shown that the isolated $\mathrm{C}$-terminal domain (C-terminal fragment) is folded in a stable defined three-dimensional structure $(5,6)$. Its secondary structure has been determined by NMR methods. It contains a flexible $\mathrm{N}$-terminal end of 10 residues, followed by three $\alpha$-helices and four $\beta$-strands (7). Sequence comparisons have shown that this domain belongs to a large family of RNA binding domains (8). The structures of two representative members of this family, the heat shock protein Hsp15 and the ribosomal protein $\mathrm{S} 4$, have been solved $(9-11)$. The C-terminal domain of TyrRS has similar structures in the 
full-length protein and as an isolated fragment (6). One can therefore assume that the apparent disorder of the C-terminal domain in the crystals of the full-length TyrRS is due to the flexibility of the polypeptide segment that links the $\mathrm{N}$ - and C-terminal domains and that TyrRS has not evolved strong noncovalent interactions between its catalytic and anticodon binding domains, contrary to the other synthetases (6).

Protein engineering experiments have shown that the acceptor arm of tRNA $^{\text {Tyr }}$ interacts with the N-terminal domain of one TyrRS subunit whereas its anticodon arm interacts with the C-terminal domain of the other subunit $(12,13)$. Residues of TyrRS that interact with $\mathrm{tRNA}^{\mathrm{Tyr}}$ have been identified by mutagenesis $(13,14)$. In addition, residue Glu152 prevents interactions of TyrRS with noncognate tRNAs without being involved in the recognition of tRNA ${ }^{\mathrm{Tyr}}$ $(15,16)$.

In the present work, we studied the structural and functional properties of the polypeptide segment, which is located at the junction between the $\mathrm{N}$ - and C-terminal domains of TyrRS. We increased its length and flexibility by introducing Gly residues; we rigidified it by introducing a Pro residue; we determined the importance of its sequence by mutating some of its residues into Ala; finally, we dissected the functional role of Phe323 by making multiple mutations of this residue. The functional effects of the mutations were tested by tRNA charging assays. The results strongly suggest that Phe323 belongs to the $\alpha$-helical subdomain of TyrRS, that it is involved in the specific recognition of the anticodon arm of $\mathrm{tRNA}^{\mathrm{Tyr}}$, and that the $\alpha$-helical subdomain is linked to the ordered C-terminal domain by a flexible peptide segment.

\section{MATERIALS AND METHODS}

Sequence Analysis. The sequences of the TyrRSs from various organisms were retrieved by using the DBGET database (http://www.genome.ad.jp/dbget-bin/www_bfind?protein-today) (17) and by entering the key word "tyrosyl tRNA synthetase" into the search box in "bfind" mode. The sequences were aligned with the Clustal W program (version 1.74) (18).

Media and Buffers. The LB, 2YT, and minimal media have been described (19). Ampicillin was used at $100 \mu \mathrm{g} / \mathrm{mL}$ and isopropyl $\beta$-D-thiogalactoside (IPTG) at $0.1 \mathrm{mM}$. The cultures were grown at $37{ }^{\circ} \mathrm{C}$. The following buffers were used: buffer A, $20 \mathrm{mM}$ Tris-HCl, pH 7.9, and $0.5 \mathrm{M} \mathrm{NaCl}$; buffer B, $50 \mathrm{mM}$ Tris- $\mathrm{HCl}$, pH 7.5, $10 \mathrm{mM}$ 2-mercaptoethanol, and $0.1 \mathrm{mM}$ phenylmethanesulfonyl fluoride (PMSF); buffer $\mathrm{C}$, $100 \mathrm{mM}$ Tris-HCl, $44 \mathrm{mM}$ Tris (pH 7.78), 10 mM 2-mercaptoethanol, and $0.1 \mathrm{mM}$ PMSF; standard buffer, $10 \mathrm{mM}$ $\mathrm{MgCl}_{2}$ (free) in buffer C. Additional $\mathrm{MgCl}_{2}$ was added to the standard buffer where necessary to compensate for its complexing with ATP.

Bacterial Strains and Parental Plasmids. The Escherichia coli K12 strains TG2, BL21(DE3) (19), and RZ1032 (20) and plasmid pVG1 (6) have been described. pVG1 carries a mutant allele of the tyrS gene from B. stearothermophilus, tyrS(BsmI, His6), under control of a promoter for the RNA polymerase of phage T7. This allele contains a change of GCG (Ala) into TGC (Cys) at codon position 321 of the tyr $S$ gene, which introduces a $B s m I$ restriction site; it also contains a $3^{\prime}$-terminal extension, 5' CTC GAG (CAC) ${ }_{6}$ TGA $3^{\prime}$, coding for the octapeptide N-Leu-Glu-His ${ }_{6}-\mathrm{C}$. We used strain TG2, in which the derivatives of the tyrS gene were not expressed, as a cellular host for all of the intermediate genetic constructions. We used strain BL21(DE3) as a cellular host to express the derivatives of the tyrS gene that were carried by plasmid pVG1 and its derivatives.

Construction of the Mutations. Oligonucleotide sitedirected mutagenesis (20) and the insertion of doublestranded DNA cassettes within or between restriction sites (6) were performed as described. Long restriction fragments were separated from short ones ( $\leq 60$ base pairs) with a QIAquick Spin column (QIAgen). All the genetic constructions were derived from plasmid pVG1. An EcoRV restriction site was created at the level of codons 326-327 (GACATT) of the tyrS(BsmI, His6) allele by mutagenesis of pVG1 with oligonucleotide 5' TGT CAA ATT GGC GAT ATC GCC GCT AAA GA 3' (the mutant codons are in italics). We called the resultant plasmid pCG1. An AflII site was created at the level of codons 339-340 (TTC-AAA) of tyrS(BsmI, His6) by mutagenesis of pVG1 with oligonucleotide 5' TGA CGG TAC ATC CTT AAG CCC TTG CTC AAT $3^{\prime}$, resulting in plasmid $\mathrm{pCG} 2$. The other mutations were constructed by the insertion of double-stranded DNA cassettes within the BsmI site of pVG1, between the BsmI and $E c o$ RV sites of pCG1, or between the BsmI and AflII sites of pCG2 (Table 1). The presence of the mutations was verified by DNA sequencing.

Preparations of Proteins. Strain BL21(DE3, pVG1) and its mutant derivatives were grown overnight in $2 \times$ YT broth with ampicillin. The cell suspension was diluted in the same medium to a starting $A_{600 \mathrm{~nm}}=0.10-0.15$ and grown under the same conditions. The cells were induced with IPTG at $A_{600 \mathrm{~nm}}=0.7$, further grown during $4 \mathrm{~h}$, and then harvested by centrifugation. Soluble cellular extracts were prepared as described (21), except that an additional step was added. The extracts were heated during $30 \mathrm{~min}$ at $58{ }^{\circ} \mathrm{C}$ to precipitate the endogenous $E$. coli TyrRS and then centrifuged for 10 min at $14000 \mathrm{~g}$ to eliminate the protein precipitate. They were used immediately for active site titrations and tRNA charging assays. The recombinant TyrRSs were purified essentially as described (6). In particular, the cell pellet from a $500 \mathrm{~mL}$ culture was resuspended in $20 \mathrm{~mL}$ of $5 \mathrm{mM}$ imidazole in buffer A, and the bacterial cells were disrupted by sonication. The proteins were separated on a column of nickel chelation resin ( $2 \mathrm{~mL}$, His-bind, Novagen), and $1 \mathrm{~mL}$ fractions were collected during the elution with imidazole. The fractions were analyzed by electrophoresis through polyacrylamide gels. The fractions with $>95 \%$ purity were pooled and dialyzed for $4 \mathrm{~h}$ against $0.1 \mathrm{mM}$ tetrasodium pyrophosphate in buffer B to remove any enzyme-bound Tyr-AMP, for $4 \mathrm{~h}$ against buffer B, and then overnight against buffer $\mathrm{C}$. The purified enzyme aliquots were snap frozen in liquid nitrogen and stored at $-70{ }^{\circ} \mathrm{C}$. Previous studies have shown that the endogeneous TyrRS of the producing cells does not copurify with the recombinant TyrRS from B. stearothermophilus when the purification is performed as described above. This conclusion was based on the composition in amino acids of the purified TyrRSs, obtained by chemical analysis, on their mass spectra (6), and on the lack of tRNA ${ }^{\text {Tyr }}$ charging by purified preparations of inactive mutant TyrRSs (V. Guez, unpublished). 


\begin{tabular}{|c|c|c|c|}
\hline mutation & DNA cassette ${ }^{a}$ & ends ${ }^{b}$ & $\operatorname{site}^{c}$ \\
\hline Y2 & $\begin{array}{l}+5^{\prime} \text { TGggaggcC } 3^{\prime} \\
-5^{\prime} \text { cetccCAGG } 3^{\prime}\end{array}$ & BsmI, BsmI & $+S t u \mathrm{I}$ \\
\hline Y3 & $\begin{array}{l}+5^{\prime} \text { TGggcggaggcC } 3^{\prime} \\
-5^{\prime} \text { cctccgccCAGG } 3^{\prime}\end{array}$ & BsmI , BsmI & $+S t u \mathrm{I}$ \\
\hline Y4 & $\begin{array}{l}+5^{\prime} \text { ttttctcaggcC } 3^{\prime} \\
-5^{\prime} \text { cctgagaaaaGG } 3^{\prime}\end{array}$ & BsmI, BsmI & $+S t u \mathrm{I}$ \\
\hline Y5 & $\begin{array}{l}+5^{\prime} \text { tttctcaggaggcC } 3^{\prime} \\
-5^{\prime} \text { cctcctgagaaaaGG } 3^{\prime}\end{array}$ & BsmI, BsmI & $+S t u \mathrm{I}$ \\
\hline Y6 & $\begin{array}{l}+5^{\prime} \text { tttctcaggcggaggcC } 3^{\prime} \\
-5^{\prime} \text { cctccgectgagaaaaGG } 3^{\prime}\end{array}$ & BsmI , BsmI & $+S t u \mathrm{I}$ \\
\hline Y7 & $\begin{array}{l}+5^{\prime} \text { TCTTTTCCGGAggcGAT } 3^{\prime} \\
-5^{\prime} \text { ATCgccTCCGGAAAAGAGG 3' }\end{array}$ & BsmI, EcoRV & $+B s p \mathrm{EI}$ \\
\hline Y8 & $\begin{array}{l}+5^{\prime} \text { TCTTTTCCGGAggtggcGAT } 3^{\prime} \\
-5^{\prime} \text { ATCgccaccTCCGGAAAAGAGG 3' }\end{array}$ & BsmI, EcoRV & $+B s p \mathrm{EI}$ \\
\hline Y9 & $\begin{array}{l}+5^{\prime} \text { TCTTTTCCGGAggcggtggcGAT } 3^{\prime} \\
-5^{\prime} \text { ATCgccaccgccTCCGGAAAAGAGG 3' }\end{array}$ & BsmI, EcoRV & $+B s p \mathrm{EI}$ \\
\hline Y10 & $\begin{array}{l}+5^{\prime} \text { TCTTTAGCGGCGACATTGCCAATT 3' } \\
-5^{\prime} \text { TTAAAATTGGCAATGTCGCCGCTAAAGAGG 3' }\end{array}$ & BsmI, AflII & $-A f l \mathrm{II}$ \\
\hline L322P & $\begin{array}{l}+5^{\prime} \text { cgTTTAGCGGCGAT 3' } \\
-5^{\prime} \text { ATCGCCGCTAAAcggG 3' }\end{array}$ & BsmI, EcoRV & none \\
\hline F323A & $\begin{array}{l}+5^{\prime} \text { TCgcgAGCGGCGAT 3' } \\
-5^{\prime} \text { ATCGCCGCTcgcGAGG 3' }\end{array}$ & BsmI, EcoRV & $+N r u \mathrm{I}$ \\
\hline F323L & $\begin{array}{l}+5^{\prime} \text { TCttaAGCGGCGAT } 3^{\prime} \\
-5^{\prime} \text { ATCGCCGCTtaaGAGG } 3^{\prime}\end{array}$ & BsmI, EcoRV & $+A f l I I$ \\
\hline F323Y & $\begin{array}{l}+5^{\prime} \text { TCtatAGCGGCGAT } 3^{\prime} \\
-5^{\prime} \text { ATCGCCGCTataGAGG 3' }\end{array}$ & BsmI, EcoRV & $+S f c \mathrm{I}$ \\
\hline F323W & $\begin{array}{l}+5^{\prime} \text { TCtggAGCGGCGAT } 3^{\prime} \\
-5^{\prime} \text { ATCGCCGCTccaGAGG 3' }\end{array}$ & BsmI, EcoRV & $+B p m \mathrm{I}$ \\
\hline S324A & $\begin{array}{l}+5^{\prime} \text { TCTTTgccGGCGAT 3' } \\
-5^{\prime} \text { ATCGCCggcAAAGAGG 3' }\end{array}$ & BsmI, EcoRV & $+N a e \mathrm{I}$ \\
\hline G325A & $\begin{array}{l}+5^{\prime} \text { TCTTTAGCgctGAT } 3^{\prime} \\
-5^{\prime} \text { ATCagcGCTAAAGAGG } 3^{\prime}\end{array}$ & BsmI, EcoRV & none \\
\hline
\end{tabular}

${ }^{a}$ Sequences of the sense $(+)$ and antisense $(-)$ strands of the cassette; the nucleotides added or modified by the cassette are in lower case letters. ${ }^{b}$ Restriction sites at the ends of the cassette. ${ }^{c}$ Restriction site added $(+)$ or removed $(-)$ by the mutation.

Active Site Titration. The concentration of TyrRS was measured by active site titration at $25^{\circ} \mathrm{C}$ in standard buffer, essentially as described (22). For pure preparations of TyrRS, the reaction mixture $(200 \mu \mathrm{L})$ contained $20 \mu \mathrm{M}\left[{ }^{14} \mathrm{C}\right] \mathrm{Tyr}, 2$ $\mathrm{mM}$ ATP $\cdot \mathrm{Mg}^{2+}, 10$ units $/ \mathrm{mL}$ inorganic pyrophosphatase (from baker's yeast, Sigma), and $0.25-2.5 \mu \mathrm{M}$ pure enzyme. Aliquots were spotted onto nitrocellulose filters at 1, 3, 5, 10 , and $20 \mathrm{~min}$ and then washed with $25 \mathrm{~mL}$ of ice-cold, five times diluted standard buffer. For soluble extracts of TyrRS producing cells, the reaction mixture $(60 \mu \mathrm{L})$ contained $20 \mu \mathrm{M}\left[{ }^{14} \mathrm{C}\right] \mathrm{Tyr}, 10 \mathrm{mM} \mathrm{ATP} \cdot \mathrm{Mg}^{2+}$, and $32 \mu \mathrm{L}$ of soluble extract. It was incubated for $2 \mathrm{~min}$ in the presence of $1 \mathrm{mM}$ sodium pyrophosphate to displace unlabeled TyrAMP from TyrRS. Pyrophosphate was then hydrolyzed with pyrophosphatase (10 units/mL) to initiate the formation of ${ }^{14} \mathrm{C}$-labeled Tyr-AMP. Aliquots were spotted onto nitrocellulose filters after 5 and $30 \mathrm{~min}$ and washed as described above.

Kinetic Procedures. The kinetic assays were performed at $25{ }^{\circ} \mathrm{C}$ in standard buffer, essentially as described (22). The pyrophosphate exchange reaction was performed in 2 $\mathrm{mM}$ ATP $\cdot \mathrm{Mg}^{2+}, 50 \mu \mathrm{M}$ tyrosine, and $2 \mathrm{mM}\left[{ }^{32} \mathrm{P}\right]$ pyrophosphate. The concentration of TyrRS active sites in the reaction was equal to $100 \mathrm{nM}$. The $K_{\mathrm{M}}$ of the wild-type TyrRS in this reaction is $0.9 \mathrm{mM}$ for ATP and $2.4 \mu \mathrm{M}$ for tyrosine (22).

The initial rate of tRNA ${ }^{\text {Tyr }}$ charging with $\left[{ }^{14} \mathrm{C}\right]$ tyrosine was measured as follows. For pure preparations of TyrRS and its derivatives, the enzyme was diluted in standard buffer containing $0.1 \mathrm{mg}$ of bovine serum albumin $/ \mathrm{mL}$. The reaction mixture contained $0.1 \mathrm{mg}$ of bovine serum albumin $/ \mathrm{mL}, 10$ $\mathrm{mM}$ ATP $\cdot \mathrm{Mg}^{2+}, 20 \mu \mathrm{M}\left[{ }^{14} \mathrm{C}\right]$ tyrosine, and 10 units $/ \mathrm{mL}$ inorganic pyrophosphatase, $E$. coli $\mathrm{tRNA}^{\mathrm{Tyr}}$, and enzyme in a total volume of $125 \mu \mathrm{L}$. Pure $E$. coli tRNA $^{\mathrm{Tyr}}$ was used at concentrations between 0.1 and $6.0 \mu \mathrm{M}$. Every minute during the first $4 \mathrm{~min}, 25 \mu \mathrm{L}$ portions of the reaction were spotted onto Whatman 3MM paper disks, and after $10 \mathrm{~s}$, the disks were immersed into $5 \%(\mathrm{w} / \mathrm{v})$ trichloroacetic acid, then washed, and counted as described (15). For soluble extracts of TyrRS producing cells, pure tRNA ${ }^{\text {Tyr }}$ was replaced by 5 $\mathrm{mg} / \mathrm{mL}$ crude $E$. coli tRNA (corresponding to $1.8 \mu \mathrm{M}$ tRNA $^{\text {Tyr }}$ ). The $K_{\mathrm{M}}$ of the wild-type TyrRS in the charging reaction is $2.2 \mathrm{mM}$ for ATP and $2.0 \mu \mathrm{M}$ for tyrosine $(22$, $23)$. The charging capacity of $\mathrm{RNA}^{\mathrm{Tyr}}$ was determined with $100 \mathrm{nM}$ parental TyrRS. Pure E. coli $\mathrm{tRNA}^{\mathrm{Tyr}}$ (Subriden RNA) was at $23 \mathrm{nmol}$ of tyrosine acceptance/mg. Crude $E$. coli tRNA (a gift from John Smith, Cambridge, U.K.) was at $356 \mathrm{pmol}$ of tyrosine acceptance/mg. The kinetic parameters at steady state, $K_{\mathrm{M}}\left(\mathrm{tRNA}^{\mathrm{Tyr}}\right)$ and $k_{\text {cat }}$, were determined by fitting the Michaelis-Menten equation to the experimental values of the initial rate, using Kaleidagraph (Synergy Software).

\section{RESULTS}

Sequence of the Linking Peptide in Bacterial TyrRSs. We aligned the available sequences of bacterial TyrRSs (Figure $2)$. The alignment showed a well-conserved segment, upstream from residue position 324 in TyrRS from B. stearothermophilus (all of the numberings refer to this organism). In particular, Phe 323 was conserved in $83 \%$ of the sequences. 


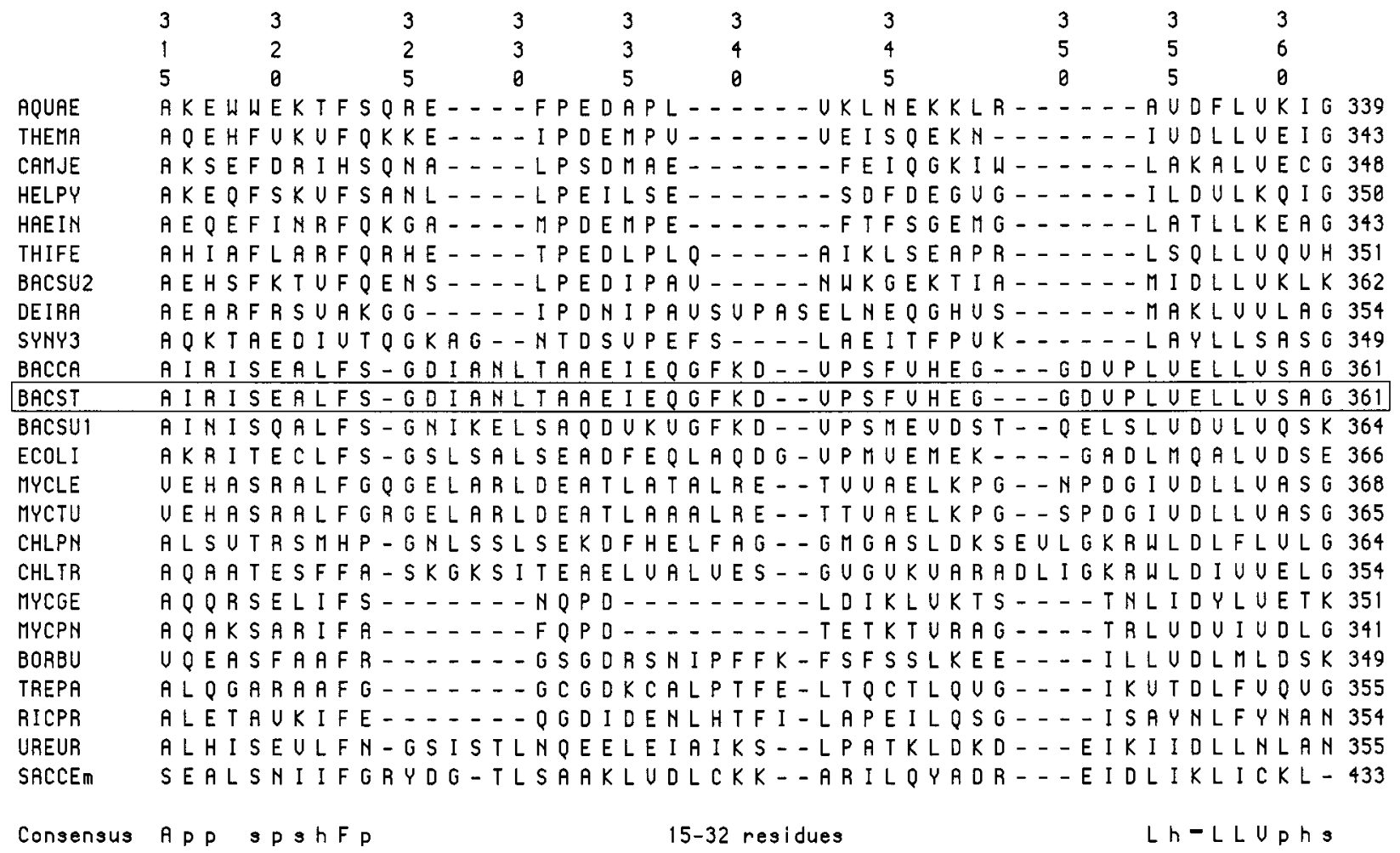

FIGURE 2: Sequence alignment of bacterial TyrRSs in the region of the linking peptide. The sequences were retrieved and aligned as described under Materials and Methods. The sequence from B. stearothermophilus is boxed. The numbering of the top line corresponds to the sequence from this organism. A $66 \%$ consensus is given in the bottom line. p, s, h, and - denote polar, small, hydrophobic, and negatively charged residues, respectively. Note the presence of the conserved Phe323 residue. Abbreviations: AQUAE, Aquifex aeolicus; BACCA, Bacillus caldotenax; BACST, B. stearothermophilus; BACSU1 and BACSU2, products of the tyrS and tyrZ genes from Bacillus subtilis, respectively; BORBU, Borrelia burgdorferi; CAMJE, Campylobacter jejuni; CHLPN, Chlamydophila pneumoniae; CHLTR, Chlamydia trachomatis; DEIRA, Deinococcus radiodurans; ECOLI, E. coli; HAEIN, Haemophilus influenzae; HELPY, Helicobacter pylori; MYCLE, Mycobacterium leprae; MYCGE, Mycoplasma genitalium; MYCPN, Mycoplasma pneumoniae; MYCTU, Mycobacterium tuberculosis; RICPR, Rickettsia prowazekii; SACCEm, Saccharomyces cerevisiae (mitochondrial); SYNY3, Synechocystis sp. (PCC 6803); THEMA, Thermotoga maritima; THIFE, Thiobacillus ferrooxidans; TREPA, Treponema pallidum; UREUR, Ureaplasma urealyticum.

The segment between positions 325 and 353 was highly variable in sequence and length. The alignement showed another very conserved segment, downstream from position 354. This segment corresponds to the beginning of a domain which is present in a numerous family of RNA binding proteins (see introduction).

Construction and Production of the Mutant TyrRSs. To test the functional importance of the residues identified by sequence analysis, we constructed mutations at the corresponding positions in TyrRS from B. stearothermophilus. All of the mutant TyrRS derived from the same parental enzyme, which was encoded by plasmid pVG1, was called TyrRS* and carried mutation A321C and a C-terminal extension Leu-Glu-His 6 . Mutation A321C, which results from the introduction of a $B s m I$ site in the tyrS gene, and the eightresidue extension, which facilitates the purification of TyrRS, affect only weakly its activity of tRNA ${ }^{\text {Tyr }}$ charging with tyrosine (see below; 6, 24). The yields of the TyrRS* derivatives, measured by titration of their active sites in soluble extracts of producing cells, were high and varied between 0.14 and 2.7 times that of the parental TyrRS*. We observed that the yield of the TyrRS* derivatives was roughly a decreasing function of their specific activity. This observation can be explained by the fact that TyrRS is toxic when overproduced in E. coli (21).

Activity of the TyrRS Derivatives in the Charging of tRNA. The structure of TyrRS from B. stearothermophilus becomes disordered after residue Ser319, and yet this sequence remains conserved, among the bacterial TyrRSs, down to Phe323. To determine the functional importance of the length and flexibility of segment Glu320-Phe323, we constructed mutations Y2 and Y3, which inserted two or three Gly residues, respectively, between Leu322 and Phe323 and the point mutation L322P. The $\phi$ and $\psi$ dihedral angles of a Gly residue can occupy most of the Ramachandran plane and therefore allow the polypeptide chain a large conformational freedom. In contrast, those of Pro take defined values and constrain conformation (25). We found that Y2, $\mathrm{Y} 3$, and L322P decreased the specific activity of TyrRS* in the charging of tRNA ${ }^{\mathrm{Tyr}}$ with tyrosine 11-, 50-, and 50-fold, respectively (Table 2 ). Thus, the length and conformation of segment Glu320-Phe323 were important for the function of TyrRS.

Phe323, Ser324, and Gly325 belong to the border between the conserved and variable segments of the linker peptide. To define precisely this border and the role of these three residues, we changed them into Ala. Mutation F323A decreased the specific activity of TyrRS* in tRNA ${ }^{\text {Tyr }}$ charging 90-fold, whereas S324A and G325A only slightly increased it, 2- and 5-fold, respectively (Table 2). Thus, the side chain of Phe323 was essential for charging, but the $\mathrm{O} \gamma$-H group of Ser324 and the lack of a side chain in position 325 were not important. We then constructed mutations $\mathrm{F} 323 \mathrm{~W}$, F323Y, and F323L, in addition to F323A, to analyze which 


\begin{tabular}{|c|c|c|}
\hline mutation & sequence change ${ }^{b}$ & $\begin{array}{c}\text { specific } \\
\text { activity }^{c}\left(\mathrm{~s}^{-1}\right)\end{array}$ \\
\hline parent & C321-L322-F323-S324-G325-D326 & $2.0 \pm 0.3$ \\
\hline EcoRV & none & $2.5 \pm 1.1$ \\
\hline AflII & G338-L-K340 & $2.1 \pm 0.5$ \\
\hline Y2 & L322-GGL-F323 & $0.18 \pm 0.03$ \\
\hline Y3 & L322-GGGL-F323 & $0.04 \pm 0.01$ \\
\hline Y4 & G325-LFSG-D326 & $1.9 \pm 0.3$ \\
\hline Y5 & G325-GLFSG-D326 & $1.7 \pm 0.1$ \\
\hline Y6 & G325-GGLFSG-D326 & $1.8 \pm 0.3$ \\
\hline Y7 & G325-G-D326 & $1.04 \pm 0.07$ \\
\hline Y8 & G325-GG-D326 & $1.1 \pm 0.2$ \\
\hline Y9 & G325-GGG-D326 & $2.0 \pm 0.1$ \\
\hline $\mathrm{Y} 10$ & N329-F339 & $0.007 \pm 0.005$ \\
\hline L322P & C321-P-F323 & $0.04 \pm 0.02$ \\
\hline F323A & L322-A-S324 & $0.022 \pm 0.005$ \\
\hline F323L & L322-L-S324 & $0.03 \pm 0.01$ \\
\hline F323Y & L322-Y-S324 & $0.6 \pm 0.2$ \\
\hline F323W & L322-W-S324 & $1.97 \pm 0.07$ \\
\hline S324A & F323-A-G325 & $4.0 \pm 2.1$ \\
\hline G325A & S324-A-D326 & $10.2 \pm 3.5$ \\
\hline
\end{tabular}

${ }^{a}$ Experimental conditions given in Materials and Methods; $25^{\circ} \mathrm{C}$ in standard $\mathrm{pH} 7.78$ buffer, with $10 \mathrm{mM}$ ATP $\cdot \mathrm{Mg}^{2+}, 20 \mu \mathrm{M}$ tyrosine, $10 \mathrm{mM} \mathrm{MgCl}_{2}$, and $5 \mathrm{mg} / \mathrm{mL}$ crude $E$. coli tRNA (356 pmol of tyrosine acceptance/mg). Specific activities are quoted per mole of dimeric enzyme, as measured by active site titration. ${ }^{b}$ The first row give the parental sequence. The other rows give the changes or insertions of residues between dashes; the flanking sequences remained identical to the parental ones. ${ }^{c}$ Means and standard errors from three experiments.

specific properties of the Phe323 side chain were essential. We found that the relative values of the specific activity were equal to 100 for Phe as in the parental TyrRS*, 99 for Trp, 30 for Tyr, 1.5 for Leu, and 1.1 for Ala (Table 2). Thus, the aromaticity of the side chain in position 323 was necessary for charging.

To test the functional importance of the segment which is nonconserved and located downstream from residue Gly325, we constructed mutations $\mathrm{Y} 4$ to $\mathrm{Y} 9$, which inserted from 1 to 6 residues (in particular Gly) between Gly325 and Asp326. We also constructed Y10, which precisely deleted Leu330Gly338. We found that insertions Y4-Y9 decreased the specific activity of TyrRS* in tRNA ${ }^{\text {Tyr }}$ charging less than 2-fold, whereas deletion Y10 decreased it 285-fold (Table 2). Therefore, the segment downstream from Gly325 could be lengthened and made more flexible without consequence on the activity of TyrRS. In contrast, segment 330-338, which folds into helix $\mathrm{H}_{2}$ ", was indispensable in $B$. stearothermophilus even though it is very variable between organisms or even absent in some of them (Figure 2) (7). Mutation F339L, which resulted from the introduction of an AflII site in the tyrS gene, had no effect on the specific activity of TyrRS* in tRNA ${ }^{\text {Tyr }}$ charging (Table 2).

Kinetics of $t R N A^{T y r}$ Charging by the Phe 323 Mutants. The above results showed that residue Phe323 was involved in the charging of tRNA $^{\text {Tyr }}$ by TyrRS. To obtain further information on its role, we performed steady-state kinetics experiments with purified preparations of the parental enzyme, TyrRS*, and of the four derivatives which carried mutations F323A, F323L, F323Y, and F323W. The specific activity of TyrRS* in the pyrophosphate exchange reaction was measured at high concentrations of substrates $(50 \mu \mathrm{M}$ tyrosine, $2 \mathrm{mM}$ ATP, and $2 \mathrm{mM}$ pyrophosphate). It was equal to $5 \mathrm{~s}^{-1}$ and not modified by the mutations. Thus, the
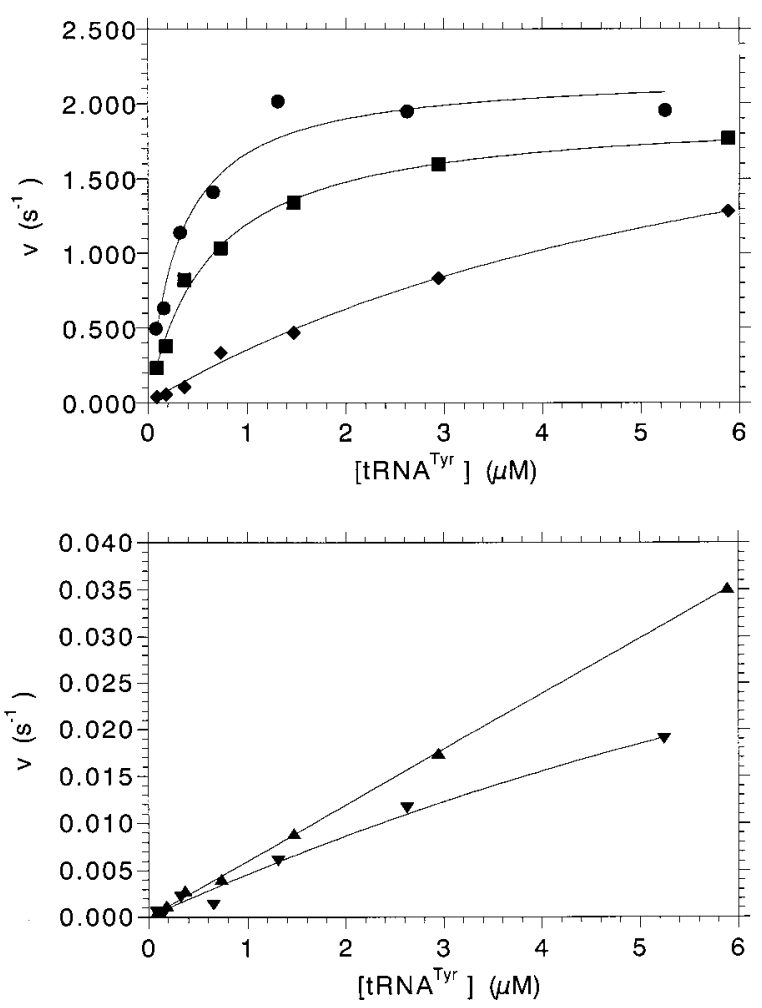

FIGURE 3: Representative Michaelis-Menten plots for the charging of $E$. coli $\mathrm{tRNA}^{\mathrm{Tyr}}$ by TyrRS* derivatives. Conditions are as in Table 3. Symbols: 0 , wild-type TyrRS*; $\mathbf{0}$, F323W mutant; $\bullet$, F323Y mutant; $\boldsymbol{\Delta}$, F323L mutant; $\mathbf{\nabla}$, F323A mutant.

\begin{tabular}{|c|c|c|c|}
\hline mutation & $\begin{array}{l}k_{\text {cat }} \\
\left(\mathrm{s}^{-1}\right)\end{array}$ & $\begin{array}{c}K_{\mathrm{M}} \\
(\mu \mathrm{M})\end{array}$ & $\begin{array}{c}k_{\text {cat }} / K_{\mathrm{M}} \\
\left(\mathrm{s}^{-1} \cdot \mathrm{mM}^{-1}\right)\end{array}$ \\
\hline parental & $1.9 \pm 0.2$ & $0.28 \pm 0.03$ & $6965 \pm 304$ \\
\hline F323A & $0.047 \pm 0.006$ & $7.9 \pm 1.6$ & $6.3 \pm 0.6$ \\
\hline F323L & ND & ND & $7.4 \pm 0.7$ \\
\hline F323Y & $3.4 \pm 0.4$ & $8.9 \pm 1.2$ & $388 \pm 42$ \\
\hline F $323 W$ & $1.9 \pm 0.2$ & $0.9 \pm 0.2$ & $2255 \pm 444$ \\
\hline
\end{tabular}

${ }^{a}$ Experimental conditions given in Materials and Methods; $25^{\circ} \mathrm{C}$ in standard $\mathrm{pH} 7.78$ buffer, with $10 \mathrm{mM}$ ATP $\cdot \mathrm{Mg}^{2+}, 20 \mu \mathrm{M}$ tyrosine, $10 \mathrm{mM} \mathrm{MgCl}_{2}$, and $0.1-6 \mu \mathrm{M}$ purified $E$. coli $\mathrm{tRNA}^{\mathrm{Tyr}}(23 \mathrm{nmol}$ of tyrosine acceptance $/ \mathrm{mg}$ ). Rate constants are quoted per mole of dimeric enzyme, as measured by active site titration. Results are means and standard errors from three experiments. ND, not determined because the value of $K_{\mathrm{M}}$ was too high.

mutations of Phe323 did not affect the first step of the aminoacylation reaction under these experimental conditions. The kinetic parameters for the charging of E. coli tRNA ${ }^{\mathrm{Ty}}$ were measured at saturating concentrations of tyrosine and ATP (Figure 3, Table 3). They were close but significantly different for the parental enzyme used in this work, TyrRS*, and for the wild-type enzyme, TyrRS: the $K_{\mathrm{M}}\left(\mathrm{tRNA}^{\mathrm{Tyr}}\right)$ values were respectively equal to $0.28 \pm 0.03$ and $1.4 \pm$ $0.2 \mu \mathrm{M}^{-1}$, the $k_{\text {cat }}$ values to $1.9 \pm 0.2$ and $3.7 \pm 0.3 \mathrm{~s}^{-1}$, and the $k_{\text {cat }} / K_{\mathrm{M}}$ values to $7.0 \pm 0.3$ and $2.7 \pm 0.2 \mu \mathrm{M}^{-1} \mathrm{~s}^{-1}$ (this work; 15).

Mutations F323A and F323L increased the value of $K_{\mathrm{M}^{-}}$ $\left(\mathrm{tRNA}^{\mathrm{Tyr}}\right)$ at least 28 -fold. Therefore, the side chain of Phe323 (more precisely the distal part of its aromatic ring) contributed to the stability of the initial complex between TyrRS and tRNA ${ }^{\text {Tyr }}$. Mutation F323Y increased the value of $K_{\mathrm{M}} 32$-fold. Therefore, the hydrophobicity of the side chain in position 323 was important. Mutation F323W 
weakly increased $K_{\mathrm{M}}$, by a factor of 3 , whereas F323L strongly increased it. Therefore, not only the hydrophobicity but also the aromaticity of the side chain in position 323 were important. Thus, the stability of the initial complex depended on a precise interaction between the aromatic ring of Phe323 and tRNA ${ }^{\text {Tyr }}$.

Mutation F323A decreased the kinetic parameter $k_{\text {cat }} 41$ fold. Therefore, an interaction between the side chain of residue Phe323 and tRNA ${ }^{\text {Tyr }}$ contributed to lowering the activation energy of the initial complex between TyrRS and tRNA $^{\text {Tyr }}$. Mutations F323Y and F323W had very little effect on $k_{\text {cat }}$. Therefore, the exchange of an aromatic side chain in position 323 for any other one did not affect the activation energy.

The specificity of the interaction between tRNA ${ }^{\mathrm{Tyr}}$ and the mutant TyrRSs, given by the $k_{\mathrm{cat}} / K_{\mathrm{M}}$ parameter, was maximal when the side chain in position 323 was Phe and decreased 3-fold when it was Trp, 18-fold for Tyr, and about 1000-fold for Leu and Ala.

\section{DISCUSSION}

Reaction Step Affected by the Mutations. A priori, the mutations of this study could affect either steps of the tyrosylation reaction, i.e., the activation of tyrosine with ATP

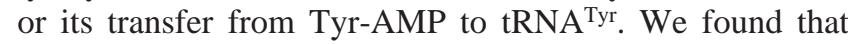
the specific activity of TyrRS* in the pyrophosphate exchange assay was not modified by the mutations of residue Phe323 in reaction conditions which were similar to those of the tRNA charging assay (see the Results section). Moreover, TyrRS $(\Delta 1)$, a truncated version of TyrRS lacking residues 318-417 and therefore Phe323, has the same kinetic parameters as the full-length TyrRS in the reactions of pyrophosphate exchange and Tyr-AMP synthesis $(26,27)$. The presence of the cognate tRNA is not necessary for the synthesis of the aminoacyl adenylate intermediate in the case of TyrRS, contrary to the case of glutaminyl-tRNA synthetase, for example. In fact, pre-steady-state kinetics have shown that the prior binding of tRNA ${ }^{\mathrm{Tyr}}$ decreases the rate of Tyr-AMP synthesis (27-29). Thus, we concluded that the mutations of the present study affected the second step of the tyrosylation reaction, i.e., the recognition of tRNA ${ }^{\mathrm{Tyr}}$ and the transfer of tyrosine from Tyr-AMP to the tRNA.

Position and Flexibility of the Linking Peptide. The changes of Leu322 into Pro and Phe323 into Ala by the point mutations L322P and F323A and the insertions of a tri- or tetrapeptide, rich in Gly, upstream from Phe323 by mutations Y2 and Y3 respectively, strongly decreased the specific activity of TyrRS* in the charging of tRNA ${ }^{\mathrm{Tyr}}$. Thus, the side chain of Phe323, its orientation, and its precise position relative to the upstream sequences were essential for the interaction of TyrRS with tRNA ${ }^{\text {Tyr }}$. In contrast, the changes of Ser324 and Gly325 by mutations S324A and G325A and the insertion of one to three residues of Gly immediately downstream from Gly325, by mutations Y7, Y8, and Y9, had little effect on the rate of charging. Thus, the nature of the residues located immediately downstream from Phe323, their conformation, and their precise number were not critical for the interaction of TyrRS with tRNA ${ }^{\mathrm{Tyr}}$. The results suggested that the wild-type sequence itself is flexible and responsible for the disorder of the $\mathrm{C}$-terminal domain in the crystals of the full-length TyrRS.
In the structure, the electronic density disappears after residue Ser319, at the C-terminal end of $\alpha$-helix H5' (1). Secondary structure predictions suggest that helix $\mathrm{H}^{\prime}$ could extend down to and include residue Ala321 (30). We found that mutations S324A and G325A increased, 2- and 5-fold, respectively, the specific activity of TyrRS* in the charging of tRNA ${ }^{\text {Tyr }}$ (Table 2). These mutations changed Ser and Gly residues, which have high probabilities of being in loop structures, into an Ala residue, which has a high probability of being in an $\alpha$-helical structure and stabilizes this type of secondary structure $(31,32)$. Mutations S324A and G325A might thus exert their effects by favoring the extension of helix $\mathrm{H}^{\prime}$ by one more turn, including residue Phe323. Our results suggest that the essential Phe 323 residue belongs to the $\alpha$-helical domain of TyrRS and that the peptide linking the $\alpha$-helical and C-terminal domains starts with residue Ser324. The deletion of residues $329-339$ by mutation Y10 abolished the charging activity of TyrRS. Therefore, the linking peptide ends in this segment of the sequence.

Phe323 Interacts with the Anticodon Arm of $t R N A^{T y r}$. As recalled above, the $\operatorname{TyrRS}(\Delta 1)$ derivative carries a deletion of residues 318-417 and yet is fully active for the formation of Tyr-AMP. Many heterodimers have been constructed between a truncated subunit of $\operatorname{TyrRS}(\Delta 1)$ and a full-length subunit of TyrRS (for a review, see ref 33). In some heterodimers, the full-length subunit carried a point mutation that prevented the formation of Tyr-AMP. These heterodimers were fully active for the charging of tRNA ${ }^{\mathrm{Tyr}}$. From these experimental data, we have deduced that the acceptor arm of tRNA ${ }^{\mathrm{Tyr}}$ interacts with the N-terminal domain of one subunit and its anticodon arm with the C-terminal domain of the other subunit (12). The same data show that Phe323, which is absent from $\operatorname{TyrRS}(\Delta 1)$, is not involved in the recognition of the acceptor arm of tRNA ${ }^{\mathrm{Tyr}}$ and that, with the C-terminal domain, it participates in the recognition of its anticodon arm.

Role of the $\alpha$-Helical Domain. No functional residue had been identified in the $\alpha$-helical domain of TyrRS up until now, and its role has remained unknown. The polypeptide loop that links the $\mathrm{C}$-terminal end of the $\alpha / \beta$ domain to the $\mathrm{N}$-terminal end of the $\alpha$-helical domain (i.e., residues $221-$ 247) contains several residues that are important for the formation of Tyr-AMP and the initial binding of tRNA ${ }^{\mathrm{Tyr}}$ $(13,34-36)$. Here, we showed that the C-terminal end of the $\alpha$-helical domain was involved in the recognition of tRNA $^{\text {Tyr }}$, through Phe 323 . Thus, the $\alpha$-helical domain serves to anchor the catalytic loop, composed of residues 221247, at the surface of TyrRS; it also serves as a spacer to position Phe323 at a distance to the active site and to enable its interaction with the anticodon arm of tRNA ${ }^{\mathrm{Tyr}}$.

Nature of the Interaction between Phe323 and tRNA ${ }^{T y r}$. Our results do not enable us to conclude whether residue Phe323 forms direct bonds with the anticodon arm of tRNA ${ }^{\text {Tyr }}$, whether it has a structural role and interacts with tRNA $^{\text {Tyr }}$ indirectly, or whether it is involved in a conformational change of TyrRS, induced by the binding of tRNA ${ }^{\text {Tyr }}$. However, the comparison of our results with published data gives some indications. Glu320 and the following residues are not visible in the electron density map of the full-length TyrRS (1). Therefore, it seems unlikely that Phe323 forms stable interactions with other residues of TyrRS and has a structural role. The mutations of Phe 323 had effects on the 
Table 4: Gibb's Free Energies of Complexes between Mutant Enzymes and tRNA ${ }^{\text {Tyr }}$ Relative to the Parental Enzyme ${ }^{a}$

\begin{tabular}{ccc}
\hline mutation & $\Delta \Delta G\left(\mathrm{kcal} \cdot \mathrm{M}^{-1}\right)$ & $\Delta \Delta G^{\ddagger}\left(\mathrm{kcal} \cdot \mathrm{M}^{-1}\right)$ \\
\hline F323A & $2.0 \pm 0.2$ & $4.2 \pm 0.1$ \\
F323L & ND & $4.1 \pm 0.1$ \\
F323Y & $2.1 \pm 0.1$ & $1.7 \pm 0.1$ \\
F323W & $0.7 \pm 0.1$ & $0.7 \pm 0.1$ \\
\hline
\end{tabular}

${ }^{a} \Delta G=-R T \ln \left(K_{\mathrm{M}}\right) ; \Delta \Delta G=\Delta G($ par $)-\Delta G($ mut $) ; \Delta G^{\ddagger}=-R T$ $\ln \left[\left(k_{\text {cat }} / K_{\mathrm{M}}\right) /(k T / h)\right] ; \Delta \Delta G^{\ddagger}=\Delta G^{\ddagger}($ mut $)-\Delta G^{\ddagger}($ par $) ;$ par, TyrRS*; mut, mutant; $R$, gas constant; $T$, temperature; $k$, Boltzmann constant; $h$, Planck constant. The standard error (SE) on $\Delta \Delta F$ (where $F$ is either G or $\mathrm{G}^{\ddagger}$ ) was calculated from the $\mathrm{SE}$ values on $\Delta F$ (mut) and $\Delta F$ (par) through the formula $[\operatorname{SE}(\Delta \Delta F)]^{2}=[\operatorname{SE}(\Delta F(\text { par }))]^{2}+[\operatorname{SE}(\Delta F(\text { mut }))]^{2}$.

charging of tRNA ${ }^{\mathrm{Tyr}}$ that depended on the exact nature of the replacing side chain (Table 3). These differences of effects were more consistent with precise interactions between Phe323 and tRNA ${ }^{\text {Tyr }}$ than with local conformational changes of TyrRS. Some data are even consistent with the appealing (but undemonstrated) hypothesis that Phe323 might interact with the anticodon of tRNA ${ }^{\mathrm{Tyr}}$. The $\mathrm{C}$-terminal end of $\alpha$-helix $\mathrm{H}^{\prime}$ is close to the anticodon in the structural model of the complex between TyrRS from B. stearothermophilus and tRNA ${ }^{\mathrm{Tyr}}(13,14,33)$. The base change U35G in the anticodon of $\mathrm{RNA}^{\mathrm{Tyr}}$ and the amino acid change F323A of TyrRS had effects on the charging of E. coli tRNA $^{\text {Tyr }}$ by TyrRS that were resemblant (37; Table 3). The respective variations of the kinetic parameters were indeed the following: 15.6- and 28.2-fold increases for $K_{\mathrm{M}}(\mathrm{tR}$ $\left.\mathrm{NA}^{\mathrm{Tyr}}\right)$; 13.2- and 40.8-fold decreases for $k_{\mathrm{cat}}$; 208- and 1108fold decreases for $k_{\text {cat }} / K_{\mathrm{M}}$. It would be interesting to study the charging of the mutant $\mathrm{tRNA}^{\mathrm{Tyr}}(\mathrm{U} 35 \mathrm{G})$ by the mutant TyrRS(F323A) and test the nonadditivity of the two mutations, as expected for a direct interaction. However, the amino acid change F323A had a more severe effect on charging than the base change U35G, so that the role of the amino acid residue Phe323 might not be simply to interact with the nucleotide residue U35.

Energetics of the Interaction between Phe323 and $t R N A^{T y r}$. The effects of the Phe 323 mutations on the free energy of interaction between TyrRS and tRNA ${ }^{\mathrm{Tyr}}$ are given in Table 4. Mutation F323A destabilized the initial complex between TyrRS and tRNA ${ }^{\text {Tyr }}$ by $2.0 \pm 0.2 \mathrm{kcal} / \mathrm{mol}$ and their transition state complex by $4.2 \pm 0.1 \mathrm{kcal} / \mathrm{mol}$. Thus, the side chain of Phe323 stabilized more the transition state complex between TyrRS and tRNA ${ }^{\text {Tyr }}$ than their initial complex, even though it is located more than $65 \AA$ from the active site of the synthetase (3). Mutations F323A and F323L destabilized the transition state complex by the same energy, $4.0 \mathrm{kcal} /$ mol. This result showed that the distal part of the aromatic ring of Phe 323 is involved in the stabilization of this complex.

Mutations F323Y and F323W destabilized the initial complex and the transition state complex between TyrRS and $\mathrm{RNNA}^{\mathrm{Tyr}}$ by the same energy, $2.0 \mathrm{kcal} / \mathrm{mol}$ for F323Y and $0.7 \mathrm{kcal} / \mathrm{mol}$ for $\mathrm{F} 323 \mathrm{~W}$. How can we explain that F323Y and F323W destabilized the two complexes by the same energy, when F323A destabilized more the transition state complex than the initial complex? Mutations F323Y and F323W could have only local effects, whereas F323A could have effects at a distance, communicated to the active site through either the tRNA or the synthetase. This hypothesis could be tested by comparing the effects of mutation F323A on the aminoacylations of the full-length tRNA $^{\text {Tyr }}$ and of a minihelix ${ }^{\text {Tyr }}$, mimicking the acceptor stem (38). Alternatively, it could be tested by constructing double mutant derivatives of TyrRS, carrying a mutation of residue Phe 323 and a mutation in the catalytic site of the enzyme, and by comparing the effects of the double mutation with those of the single mutations.

\section{CONCLUSIONS}

Our results revealed the presence of a functionally important residue in a region of TyrRS that had not been studied previously: the crystal structure of the $\mathrm{N}$-terminal domain stops after residue 319 , and the C-terminal fragment is only visible downstream from residue 330 in the NMR structure $(1,7)$. Residue Phe323, which is located at the C-terminal end of the $\alpha$-helical domain, thus extends the list of the TyrRS residues that have been identified by mutagenesis and are involved in the recognition of tRNA ${ }^{\mathrm{Tyr}}$. Seven residues of the $\alpha / \beta$ domain participate in the recognition of the acceptor stem: Thr17, Asn146, Lys151, Glu152, Trp196, Arg207, and Lys208. Six residues of the C-terminal domain participate in the interaction with either the anticodon arm or the extra arm of tRNA ${ }^{\text {Tyr: }}$ Arg368, Arg371, Arg407, Arg408, Lys410, and Lys411 (13-16). Therefore, the recognition of the anticodon arm could recruit residues from both the $\alpha$-helical intermediate domain and the $\alpha+\beta$ C-terminal domain.

Numerous data have shown that TyrRS is exceptional among the class I aminoacyl-tRNA synthetases since it approaches its cognate tRNA by the side of the variable loop and by the major groove of the acceptor stem $(13,14,33$, 39-44). A preliminary crystal structure of the complex between TyrRS and tRNA ${ }^{\text {Tyr }}$ from Thermus thermophilus has recently been presented at $3.5 \AA$ resolution (45). This crystal structure fully supports the above features of the complex between TyrRS and tRNA ${ }^{\text {Tyr }}$ and the conclusions of the present study. In particular, the $\mathrm{N}$ - and C-terminal domains of TyrRS do not make any noncovalent tertiary interaction in the crystal structure, and the anticodon arm of tRNA ${ }^{\mathrm{Tyr}}$ contacts both the $\alpha$-helical and C-terminal domains.

\section{ACKNOWLEDGMENT}

We thank Dr. Shamila Naïr for critical reading of the manuscript.

\section{REFERENCES}

1. Brick, P., Bhat, T. N., and Blow, D. M. (1989) J. Mol. Biol. 208, 83-98.

2. Eriani, G., Delarue, M., Poch, O., Gangloff, J., and Moras, D. (1990) Nature 347, 203-206.

3. Brick, P., and Blow, D. M. (1987) J. Mol. Biol. 194, 287297.

4. Waye, M. M. Y., Winter, G., Wilkinson, A., and Fersht, A. R. (1983) EMBO J. 2, 1827-1829.

5. Guez-Ivanier, V., and Bedouelle, H. (1996) J. Mol. Biol. 255, $110-120$.

6. Guez, V., Naïr, S., Chaffotte, A., and Bedouelle, H. (2000) Biochemistry 39, 1739-1747.

7. Pintar, A., Guez, V., Castagné, C., Bedouelle, H., and Delepierre, M. (1999) FEBS Lett. 446, 81-85.

8. Aravind, L., and Koonin E. V. (1999) J. Mol. Evol. 48, 291302. 
9. Staker, B. L., Korber, P., Bardwell, J. C., and Saper, M. A. (2000) EMBO J. 19, 749-757.

10. Davies, C., Gerstner, R. B., Draper, D. E., Ramakrishnan, V., and White, S. W. (1998) EMBO J. 17, 4545-4558.

11. Markus, M. A., Gerstner, R. B., Draper, D. E., and Torchia, D. A. (1998) EMBO J. 17, 4559-4571.

12. Carter, P., Bedouelle, H., and Winter, G. (1986) Proc. Natl. Acad. Sci. U.S.A. 83, 1189-1192.

13. Bedouelle, H., and Winter, G. (1986) Nature 320, 371-373.

14. Labouze, E., and Bedouelle, H. (1989) J. Mol. Biol. 205, 729735.

15. Vidal-Cros, A., and Bedouelle, H. (1992) J. Mol. Biol. 223, $801-810$

16. Bedouelle, H., and Nageotte, R. (1995) EMBO J. 14, 29452950.

17. Kanehisa, M. (1997) Trends Biochem. Sci. 22, 442-444.

18. Thompson, J. D., Higgins, D. G., and Gibson, T. J. (1994) Nucleic Acids Res. 22, 4673-4680.

19. Sambrook, J., Fritsch, E. F., and Maniatis, T. (1989) Molecular cloning: a laboratory manual, Cold Spring Harbor Laboratory Press, Cold Spring Harbor, NY.

20. Kunkel, T. A., Roberts, J. D., and Zakour, R. A. (1987) Methods Enzymol. 154, 367-382.

21. Bedouelle, H., Guez, V., Vidal-Cros, A., and Hermann, M. (1990) J. Bacteriol. 172, 3940-3945.

22. Wilkinson, A. J., Fersht, A. R., Blow, D. M., and Winter, G. (1983) Biochemistry 22, 3581-3586.

23. Ward, W. H., and Fersht A. R. (1988) Biochemistry 27, 55255530 .

24. Guez-Ivanier, V., Hermann, M., Baldwin, D., and Bedouelle, H. (1993) J. Mol. Biol. 234, 209-221.

25. MacArthur, M. W., and Thornton, J. M. (1991) J. Mol. Biol. 218, 397-412.

26. Wells, T. N., and Fersht, A. R. (1986) Biochemistry 25, 18811886.

27. Ward, W. H., and Fersht, A. R. (1988) Biochemistry 27, 10411049.

28. Fersht, A. R., and Jakes, R. (1975) Biochemistry 14, 33503356.
29. Rath, V. L., Silvian, L. F., Beijer, B., Sproat, B. S., and Steitz, T. A. (1998) Structure 6, 439-449.

30. Jermutus, L., Guez, V., and Bedouelle, H. (1999) Biochimie 81, 235-244.

31. Chou, P. Y., and Fasman, G. D. (1978) Annu. Rev. Biochem. 47, 251-276.

32. Fersht, A. R., and Serrano, L. (1993) Curr. Opin. Struct. Biol. 3, 75-83.

33. Bedouelle, H., Guez-Ivanier, V., and Nageotte, R. (1993) Biochimie 75, 1099-1108.

34. Fersht, A. R., Knill-Jones, J. W., Bedouelle, H., and Winter, G. (1988) Biochemistry 27, 1581-1587.

35. First, E. A., and Fersht, A. R. (1993) Biochemistry 32, 1365813663.

36. Xin, Y., Li, W., and First, E. A. (2000) Biochemistry 39, 340347.

37. Himeno, H., Hasegawa, T., Ueda, T., Watanabe, K., and Shimizu, M. (1990) Nucleic Acids Res. 18, 6815-6819.

38. Steer, B. A., and Schimmel, P. (1999) Proc. Natl. Acad. Sci. U.S.A. 96, 13644-13649.

39. Xin, Y., Li, W., Dwyer, D. S., and First, E. A. (2000) J. Mol. Biol. 303, 287-298.

40. Lee, C. P., and RajBhandary, U. L. (1991) Proc. Natl. Acad. Sci. U.S.A. 88, 11378-11382.

41. Quinn, C. L., Toa, N., and Schimmel, P. (1995) Biochemistry 34, 12489-12495.

42. Fechter, P., Rudinger-Thirion, J., Théobald-Dietrich, A., and Giegé, R. (2000) Biochemistry 39, 1725-1733.

43. Perret, V., Florentz, C., Dreher, T., and Giegé, R. (1989) Eur. J. Biochem. 185, 331-339.

44. Caprara, M. G., Lehnert, V., Lambowitz, A. M., and Westhof, E. (1996) Cell 87, 1135-1145.

45. Tukalo, M., Yaremchuk, A., Krikliviy, I., Grotli, M., and Cusack, S. (2000) Abstract book of the 18th tRNA Workshop "tRNA 2000", p 75, Cambridge University Press, Cambridge, U.K.

BI010208C 